\title{
Hybrid Optical Sensor for Extreme Temperature Measurement in Next Generation Higher Efficiency Greener Power Plants
}

\author{
Nabeel A. Riza \\ School of Engineering \\ University College Cork \\ Cork, Ireland \\ Email:n.riza@ucc.ie \\ http://soe.ucc.ie
}

\begin{abstract}
:
Highlighted is a novel hybrid optical design physical sensor technology for extreme environments temperature measurement that combines freespace (wireless) and fiber-optics (wired), single crystal and sintered Silicon Carbide ( $\mathrm{SiC}$ ) materials, and Black-Body radiation thermometry (pyrometry) with laser interferometry to provide robust extreme temperature measurement within a gas turbine. A first generation temperature sensor has been tested in an industrial rig. The temperature probe has survived extreme temperature reaching $1600^{\circ} \mathrm{C}$ and one month of rig operations.
\end{abstract}

Key words: optical sensor, extreme temperature measurement

\begin{abstract}
Summary
The gas turbine sensor community is constantly being challenged to provide a robust extreme temperature measurement solution, in particular to operate power plants at elevated temperatures (e.g., $1600{ }^{\circ} \mathrm{C}$ gas turbine firing temperatures) to enable greener higher efficiency operation. Given these elevated temperatures, to my knowledge, no reliable commercial solution has evolved to tackle this extreme temperature and caustic environments challenge. The purpose of this paper is to summarize the work conducted by the Riza team to realize this highly desired extreme temperature sensor for gas turbines.
\end{abstract}

Accuracy, reliability, and long-life times are critical parameters for sensors measuring temperature in gas turbines of power plants. Greener high efficiency next generation power plants need gas turbines operating at super high temperatures of $1600{ }^{\circ} \mathrm{C}$ where present thermo-couple temperature probe technology fails to operate with reliable and accurate readings over long life-times. To solve this pressing problem, we introduced the concept of a new hybrid class of all-Silicon Carbide (SiC) optical sensor shown in Fig.1 where a single crystal SiC optical chip is embedded in a sintered SiC tube assembly, forming a Coefficient of Thermal Expansion (CTE) matched all-SiC front-end probe. Because SiC chip and SiC host material are CTE matched, optimal handling of extreme thermal ramps (over $1000{ }^{\circ} \mathrm{C}$ in a few seconds) and temperatures (e.g., $1600^{\circ} \mathrm{C}$ ) is possible. The tip of the probe sits in the hot gas of the combustion chamber. Light from a laser module sitting in a remote friendly environment is fed into an optical fiber that carries the laser light to the probe entry point where a freespace laser beam is generated. This beam passes through a high temperature window into a sealed $\mathrm{SiC}$ tube and the beam strikes the SiC chip. Both incoherent BB radiation light and laser light coming from the heated SiC chip is routed via the fiber-optic to an optical receiver module also sitting in the friendly zone where postprocessing via electronics is implemented.

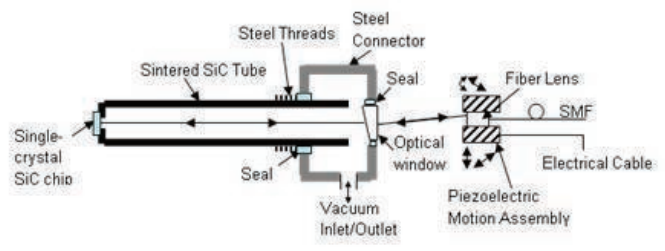

Fig. 1. Proposed basic design of the all-passive frontend all-SiC extreme temperature probe. 


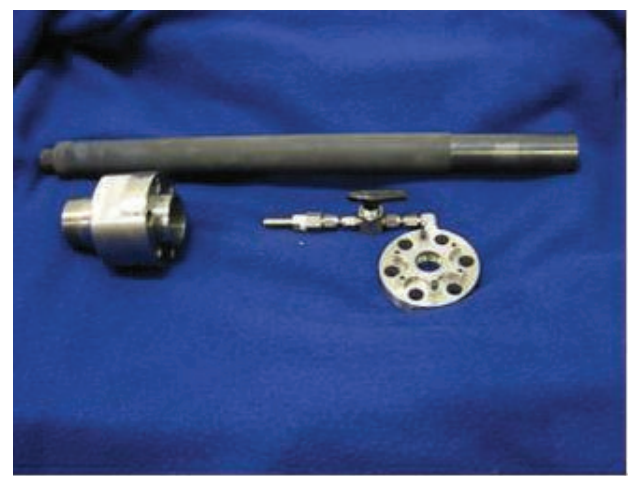

(a)

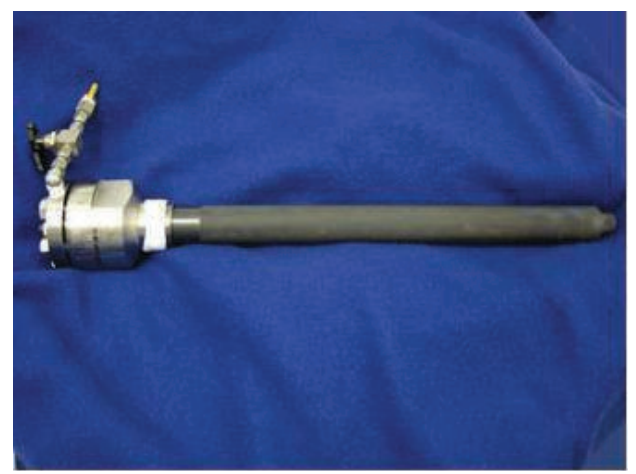

(b)

Fig.2. All-SiC temperature frontend probe shown in an (a) unassembled and (b) assembled fashion.

As shown in Fig.2, the fabricated probe consists of three connectable parts. The first part is the long (e.g., $42 \mathrm{~cm}$ ) probe that is an all-SiC hollow tube with one end open and the other end containing the embedded thick (e.g., 400 microns) SiC optical chip that optically and mechanically responds to the temperature of the gas. The second part is a steel pressure connector that engages with the open end of the probe to form a pressured sealed connection. The third part is a probe window assembly with a vacuum valve to enable a weak vacuum inside the probe cavity. The assembled probe is inserted into the gas turbine combustor inlet via a pressure fitting. A short range (e.g., $<20 \mathrm{~cm}$ ) optical transceiver module provides the laser beam for SiC chip targeting and the temperature encoded receive beam capture. Note that unlike thermo-couple and optical fiber temperature sensing approaches that require extra protection tubes around the electrical/optical wires, the proposed probe requires no such special protection; hence avoiding the CTE mismatch problems as well as the need for complex long-term packaging of many protection layers.

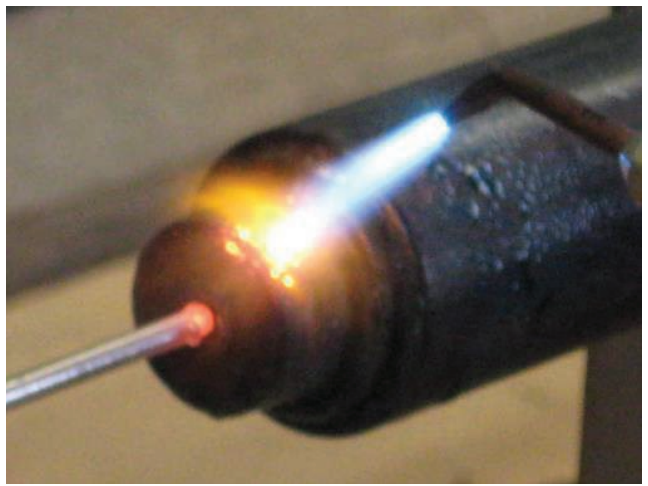

Fig.3. The All-SiC probe and a High Temperature Thermo-Couple under oxyacetylene flame thermal and localized thermal ramp joint test with temperatures reaching 1600 ${ }^{\circ} \mathrm{C}$.

Recently, a first successful industrial combustor rig test has been completed using the hybrid allSiC temperature sensor front-end probe indicating demonstrated probe structural robustness to $1600{ }^{\circ} \mathrm{C}$. Testing was carried out over a one month duration with eight combustor thermal shock tests. Fig. 3 shows a mechanical probe structural integrity test conducted with an oxy-acetylene flame where the probe remains intact but the TC melts. In order to improve SiC chip optical performance while maintaining excellent thermal heat transfer contact with the external hot gas, Fig.4 shows a preferred $\mathrm{SiC}$ chip frontend probe packaging design where the SiC chip stays protected within the sintered SiC tube housing.

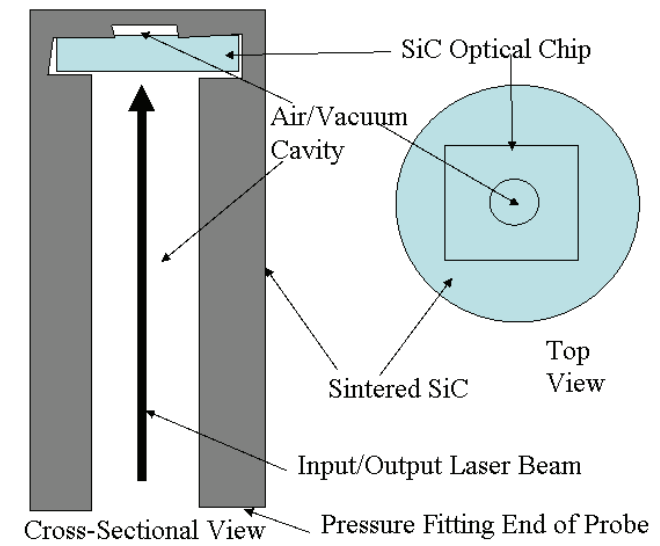

Fig.4. Proposed novel all-SiC Probe frontend design that completely protects the SiC chip from external environments that can degrade the optical performance of the chip (such as caused by dirt, soot, chemicals, etc). 


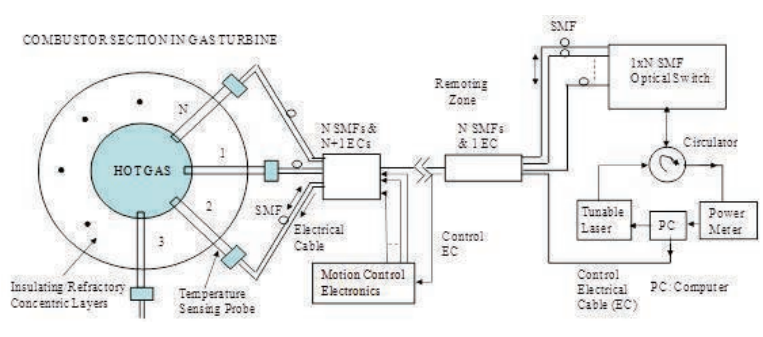

Fig.5. Proposed N temperature probes sensor network using the All-Silicon Carbide Hybrid Wireless-Wired Optics Temperature Sensors.

Fig.5 shows how the developed all-SiC temperature probe can be combined via an allfiber network for form a discrete distributed sensor network for a gas turbine. Note that all $\mathrm{N}$ probes use the same laser and detection optics and electronics, saving space, power, and cost. Further details on the proposed hybrid design $\mathrm{SiC}$ sensor can be found in the listed references [1-12].

In summary, the powers of the patented hybrid sensor design using $\mathrm{SiC}$ single crystal and sintered materials coupled with some of the best feature of fiber-optics, free-space optics, lasers, BB thermal radiation, spectral processing, optical refractive index changes, mechanical deformations, and spatial processing combine to deliver a robust temperature sensor for the gas turbine extreme environment. Future technical work consists of $\mathrm{SiC}$ probe compact design optimization and fabrication for front-end $\mathrm{SiC}$ chip long life-time operations. In addition, combined temperature plus pressure measurements is possible with modified SiC probes. Teaming with appropriate academic, national lab, and industrial partners is expected for advanced prototyping and gas engine testing.

\section{References}

[1] N. A. Riza, M. A. Arain, and F. Perez, "Harsh Environments Minimally Invasive Optical Sensor using Freespace Targeted Single Crystal Silicon Carbide," IEEE Sensors Journal, Vol. 6, June 2006.

[2] N. A. Riza and M. Sheikh, "Silicon carbide based extreme environment temperature sensor using wavelength tuned signal processing," Optics Letters, Vol.33, No.10, pp.1129-1131, May 15, 2008.

[3] M. Sheikh and N. A. Riza, "Direct Measurement High Resolution Wide Range Extreme Temperature Optical Sensor using an
all-Silicon Carbide Probe," Optics Letters, May 1, 2009.

[4] N. A. Riza and M. Sheikh, "Silicon Carbidebased High Resolution Extreme Environment Hybrid Design Temperature Sensor using Optical Pyrometry and Laser Interferometry," IEEE Sensors J., , Vol.10, No.2, Feb. 2010

[5] N. A. Riza, M. Sheikh, and F. Perez, "Hybrid Wireless-Wired Optical Sensor for Extreme Temperature Measurement in Next Generation Energy Efficient Gas Turbines," ASME Journal of Engineering for Gas Turbines and Power, vol. 132, May 2010.

[6] N. A. Riza and M. Sheikh, "All-Silicon Carbide Hybrid Wireless-Wired Optics Temperature Sensor Network Basic Design Engineering for Power Plant Gas Turbines," International Journal of Optomechatronics, March 2010.

[7] N. A. Riza and M. A. Arain, "Cryogenic Temperature Measurement using Silicon Carbide-based Wireless Optical Sensor," IEEE Photon. Tech. Lett., Vol.18, No.24, pp.25992601, Dec. 15, 2006.

[8] N. A. Riza, F. N. Ghauri, and F. Perez, "Wireless Pressure Sensor using Laser Targeting of Silicon Carbide," Optical Engineering, Vol.46, No.1, Jan. 2007.

[9] N. A. Riza, F. N. Ghauri, and F. Perez "Silicon Carbide-based remote wireless optical pressure sensor," IEEE Photon. Tech. Lett, Vol.19, April 1, 2007.

[10] N. A. Riza and F. A. Perez, "High temperature minimally invasive optical sensing modules, US Patent No. 7,327,472, Feb.5, 2008.

[11] N. A. Riza and F. Perez, "Optical sensor for extreme environments," US Patent 8,035,822, Oct.11, 2011.

[12] N. A. Riza and F. Perez, "Extreme Temperature Robust Optical Sensor Design And Fault-Tolerant Signal Processing," USA Patent 8,096,704, Jan.17, 2012. 